\title{
Lack of association between MMP13 (rs3819089), ADAM12 (rs3740199-rs1871054) and ADAMTS14 (rs4747096) genotypes and advanced-stage knee osteoarthritis
}

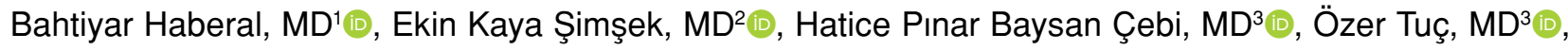 \\ Hasibe Verdi, $\mathrm{MD}^{3}{ }^{3}$, Fatma Belgin Ataç, $\mathrm{MD}^{3}{ }^{\mathbb{D}}$
}

${ }^{1}$ Department of Orthopedics and Traumatology, Başkent University, Faculty of Medicine, Ankara, Turkey

2Department of Orthopedics and Traumatology, Kaçkar State Hospital, Rize, Turkey

${ }^{3}$ Medical Biology, Başkent University, Faculty of Medicine, Ankara, Turkey

Osteoarthritis (OA) is a degenerative joint disorder associated with pain and the progressive loss of joint function, particularly the knee and hip. It is characterized by the breakdown and loss of articular cartilage due to biochemical, morphological, molecular, and biomechanical changes in the extracellular matrix (ECM) and cells. ${ }^{[1-4]}$

The multifactorial nature of $\mathrm{OA}$ has been well documented by a large number of twin-pair, sibling-risk, and segregation studies. ${ }^{[4]}$ Moreover, it is widely acknowledged that environmental and genetic factors may equally contribute to the development of OA. ${ }^{[5]}$ Furthermore, previous genome-wide association studies have found a

Received: March 10, 2021

Accepted: April 22, 2021

Published online: June 11, 2021

Correspondence: Bahtiyar Haberal, MD. Başkent Üniversitesi Tıp Fakültesi, Ortopedi ve Travmatoloji Anabilim Dalı,

06490 Bahçelievler, Ankara, Türkiye.

E-mail: bahtiyarhaberal@hotmail.com

Doi: $10.52312 /$ jdrs.2021.64

Citation: Haberal B, Kaya Şimşek E, Baysan Çebi HP, Tuç Ö, Verdi $\mathrm{H}$, Atac FB. Lack of association between MMP13 (rs3819089), ADAMI2 (rs3740199-rs1871054) and ADAMTS14 (rs4747096) genotypes and advanced-stage knee osteoarthritis. Jt Dis Relat Surg 2021;32(2):299-305.

(02021 All right reserved by the Turkish Joint Diseases Foundation

This is an open access article under the terms of the Creative Commons Attribution-NonCommercial License, which permits use, distribution and reproduction in any medium, provided the original work is properly cited and is not used for commercial purposes (http://creativecommons.org/licenses/by-nc/4.0/).

\section{ABSTRACT}

Objectives: The aim of this study was to investigate the relationship between MMP13 rs3819089, ADAM12 rs3740199 and rs1871054, and ADAMTS14 rs4747096 genotypes in patients with radiologically diagnosed knee osteoarthritis (OA).

Patients and methods: A total of 300 patients ( 68 males, 232 females; mean age: 61.6 years; range, 25 to 89 years) who were admitted to the orthopedics and traumatology clinic and diagnosed with knee OA according to the 2000 American College of Rheumatology (ACR) criteria between October 2018 and March 2019 were prospectively analyzed. Patients with Grades III-IV OA according to the KellgrenLawrence (K-L) grading system were included in the patient group $(n=150)$ and those without radiological features of knee OA (K-L Grades I-II) were included in the control group $(\mathrm{n}=150)$ voluntarily. The presence of single nucleotide polymorphisms (SNPs) in the targeted genes in both groups was assessed by real-time polymerase chain reaction in the peripheral blood sample.

Results: The most common nucleotides in both the control and patient groups were CG for $r s 3740199$ and CT for $r s 1871054$ in the ADAM12 gene, and the most common nucleotides in alleles were GG for MMP13 rs3819089 and AA for ADAMTS14 rs4747096. No statistically significant relationship was detected between the gene polymorphisms and advanced OA.

Conclusion: The study results suggest that ADAM12 rs3740199 and rs1871054, MMP13 rs3819089, and ADAMTS14 rs4747096 polymorphisms have no relationship with knee OA susceptibility in the Turkish population. However, as this is the first study to investigate the relationship between the SNPs of ADAM12, ADAMTS14, and MMP13 genes and the development of OA in the Turkish population, it would contribute to our understanding of the molecular bases of OA.

Keywords: Adam 12 protein, adamts 14 protein, knee osteoarthritis, matrix metalloproteinase 13 , single nucleotide polymorphism.

relationship with OA susceptibility. ${ }^{[3,5]}$ Therefore, the candidate gene studies may not only provide an insight into OA development, but also suggest novel therapeutic targets for improved prevention and treatment strategies. ${ }^{[5-7]}$ 
Osteoarthritic joints have an imbalance between the synthesis and degradation of ECM, which leads to cartilage destruction. Therefore, the metalloproteinase enzyme family which is responsible for ECM degradation may have a major influence on the development of OA. Previous studies have shown that single nucleotide polymorphisms (SNPs), which occur in genes with encodings of "A Disintegrin and Metalloproteinase 12" (ADAM12 rs1871054 and rs3740199), ${ }^{[4,8-12]}$ "A Disintegrin and Metalloprotease with Thrombospondin Motifs" (ADAMTS rs4747096), ${ }^{[10,12,13]}$ and "Matrix Metalloproteinases 13" (MMP13 rs3819089) $^{[14]}$ and genes that are members of the metalloproteinase enzyme family change the coding region of the gene. As a result, the function of proteins changes, and the genetic predisposition for OA appears in different populations. Moreover, ethnicity is an important factor in determining the susceptibility factors.

In the present study, we aimed to investigate the association between ADAM12 rs3740199 and rs1871054, ADAMTS14 rs4747096, and MMP13 rs3819089 genotypes in patients with radiologically diagnosed advanced-stage knee OA in the Turkish population.

\section{PATIENTS AND METHODS}

This prospective study was conducted at Başkent University Hospital Department of Orthopaedics and Traumatology between October 2018 and March 2019. The data of the patients, who were aged between 40 and 75 years and admitted to the orthopedics and traumatology clinic and diagnosed with knee OA according to the 2000 American College of Rheumatology (ACR) criteria were recorded prospectively. ${ }^{[15]}$ Direct radiographs were used for the radiological diagnoses, and the primary tibiofemoral OA was evaluated. Finally, a total of 300 patients (68 males, 232 females; mean age: 61.6 years; range, 25 to 89 years) were included. Patients with Grades III-IV OA in their knees, according to the KellgrenLawrence (K-L) grading system, who volunteered to participate in the study were included in the patient group $(n=150)$. Among the patients who applied to our clinic for any reason and had a knee radiograph, those without radiological features of advanced-stage knee OA (K-L Grades I-II) were included in the control group voluntarily $(n=150)$. Direct radiographs were evaluated independently by two orthopedics and traumatology specialists, and the consensus cases were included in the study. Patients with inflammatory arthritis, post-traumatic arthritis, septic arthritis, skeletal or developmental dysplasia, long-term hormone replacement therapy, previous knee surgery, malignancy, and a body mass index of $>25$ were excluded from the study. A written informed consent was obtained from each participant. The study protocol was approved by the Institutional Review Board and Ethics Committee (Project No: KA18/164). The study was conducted in accordance with the principles of the Declaration of Helsinki.

A total of $3 \mathrm{~mL}$ peripheral blood samples were collected in ethylenediaminetetraacetic acid (EDTA) tubes. Genomic deoxyribonucleic acid (DNA) was extracted from the peripheral blood leukocytes pellets by phenol/chloroform extraction and ethanol precipitation and, then, kept at $-20^{\circ} \mathrm{C}$ until use.

The MMP13 rs3819089, ADAM12 rs1871054 and rs3740199, and ADAMTS14 rs4747096 SNPs were determined by real-time polymerase chain reaction (PCR) testing using a panel of LightSNiP (assays based on SimpleProbe ${ }^{\circledR}$; TIB MolBiol Syntheselabor $\mathrm{GmbH}$, Berlin, Germany). The SimpleProbe ${ }^{\circledR}$ included in the LightSNP assay can detect single base mismatches, thus enabling the analysis of polymorphisms. At the end of the amplification, a melting curve analysis was performed according to the protocol (LightCycler 480, Roche, Basel, Switzerland). Polymorphic alleles were identified by the specific melting temperature (Tm) of the resulting amplicons.

For MMP13 rs3819089, individuals with two copies of the $G$ allele showed a single melting peak at $52.89^{\circ} \mathrm{C}$, individuals with the A allele showed a single melting peak at $58.38^{\circ} \mathrm{C}$, and individuals with both alleles (G/A) showed two melting peaks at $52.89^{\circ} \mathrm{C}$ and $58.38^{\circ} \mathrm{C}$ (Figure 1).

For ADAM12 rs3740199, individuals with two copies of the $\mathrm{C}$ allele showed a single melting peak at $61.28^{\circ} \mathrm{C}$, individuals with two copies of the $\mathrm{G}$ allele showed a single melting peak at $68.47^{\circ} \mathrm{C}$,

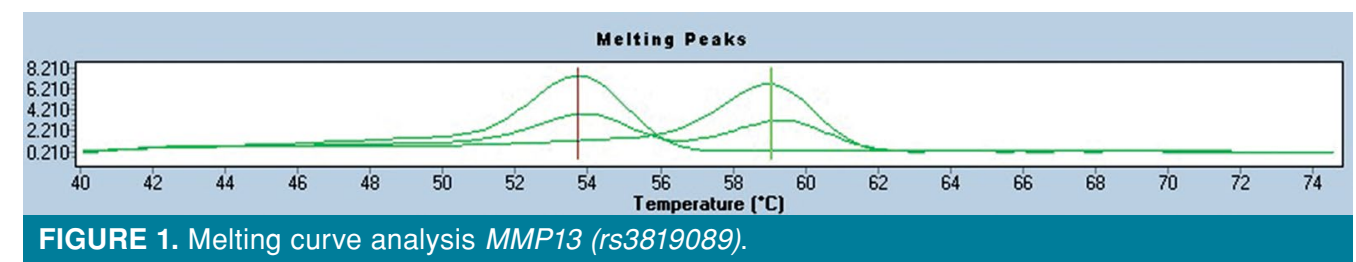




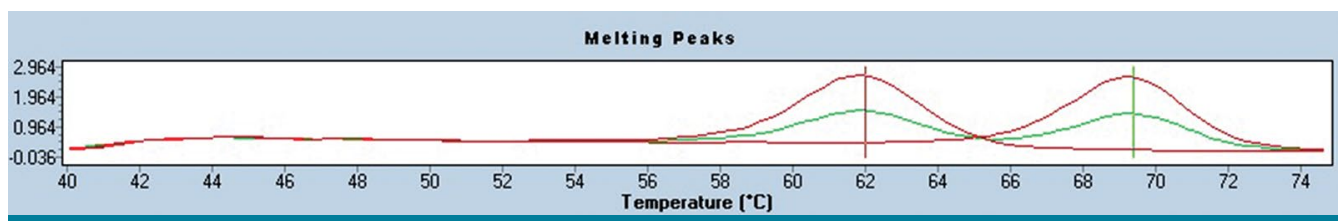

FIGURE 2. Melting curve analysis ADAM12 (rs3740199).

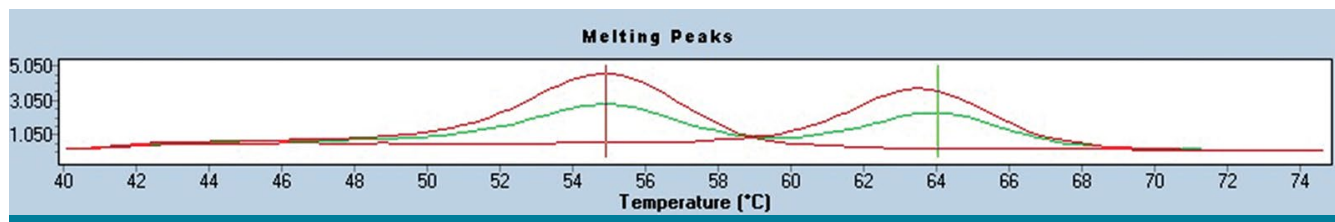

FIGURE 3. Melting curve analysis ADAMTS14 (rs4747096).

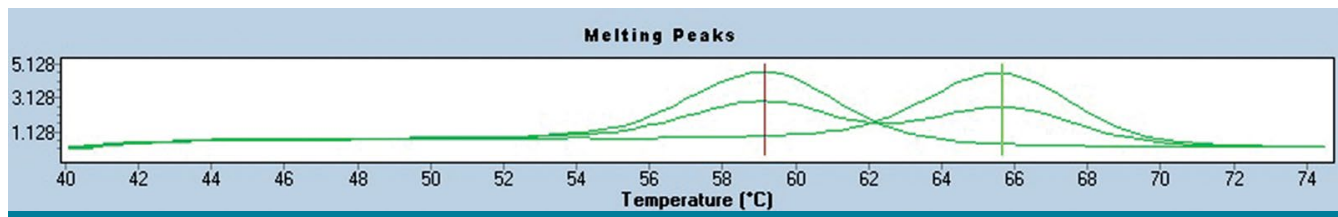

FIGURE 4. Melting curve analysis ADAM12 (rs1871054).

and individuals with both alleles $(\mathrm{C} / \mathrm{G})$ showed two melting peaks at $61.28^{\circ} \mathrm{C}$ and $68.47^{\circ} \mathrm{C}$ (Figure 2).

For ADAMTS14 rs4747096, individuals with two copies of the A allele showed a single melting peak at $53.78^{\circ} \mathrm{C}$, individuals with two copies of the $\mathrm{G}$ allele showed a single melting peak at $62.77^{\circ} \mathrm{C}$, and individuals with both alleles (A/G) showed two melting peaks at $53.78^{\circ} \mathrm{C}$ and $62.77^{\circ} \mathrm{C}$ (Figure 3).

For ADAM12 rs1871054, individuals with two copies of the $\mathrm{C}$ allele showed a single melting peak at $57.53^{\circ} \mathrm{C}$, individuals with two copies of the $\mathrm{T}$ allele showed a single melting peak at $64.18^{\circ} \mathrm{C}$, and individuals with both alleles $(\mathrm{C} / \mathrm{T})$ showed two melting peaks at $57.53^{\circ} \mathrm{C}$ and $64.18^{\circ} \mathrm{C}$ (Figure 4).

\section{Statistical analysis}

A priori power analysis was performed to calculate the sample size estimation during the project preparation phase. The sample size and power of the test were calculated using the PASS version 11.0 software (NCSS LLC, Kaysville, UT, USA). With alpha $=0.05$, beta $=0,19757$, and $W=0.2$, the power value was obtained as $0.80243(80.243 \%)$ with a sample size of 300 .

Statistical analysis was performed using the IBM SPSS for Windows version 24.0 software (IBM Corp., Armonk, NY, USA). For the univariate analysis, the chi-square test was used, and the binary logistic regression analysis and backward stepwise model

\begin{tabular}{|c|c|c|c|c|c|c|}
\hline \multicolumn{7}{|c|}{$\begin{array}{c}\text { TABLE I } \\
\text { phic characteristics of study population }\end{array}$} \\
\hline & \multicolumn{3}{|c|}{ Control Group ( $n=150)$} & \multicolumn{3}{|c|}{ Patient Group ( $n=150)$} \\
\hline & $\mathrm{n}$ & $\%$ & Mean $\pm S D$ & $\mathrm{n}$ & $\%$ & Mean \pm SD \\
\hline Age (year) & & & $57.3 \pm 11.2$ & & & $66 \pm 9.8$ \\
\hline \multicolumn{7}{|l|}{ Sex } \\
\hline Female & 108 & 72.0 & & 124 & 82.6 & \\
\hline Male & 42 & 28.0 & & 26 & 17.4 & \\
\hline \multicolumn{7}{|c|}{ K-L knee grade } \\
\hline III & - & - & & 76 & 50.6 & \\
\hline IV & - & - & & 74 & 49.4 & \\
\hline
\end{tabular}




\begin{tabular}{|c|c|c|c|c|c|}
\hline \multicolumn{6}{|c|}{$\begin{array}{c}\text { TABLE II } \\
\text { The frequencies }(\%) \text { and statistical compari }\end{array}$} \\
\hline & \multicolumn{2}{|c|}{ Control Group (KL-I/II) } & \multicolumn{2}{|c|}{ Patient Group (KL-III/IV) } & \multirow[b]{2}{*}{$p$} \\
\hline & $\mathrm{n}$ & $\%$ & $\mathrm{n}$ & $\%$ & \\
\hline \multicolumn{6}{|l|}{ ADAM12 gene } \\
\hline rs3740199 genotypes & & & & & 0.132 \\
\hline $\mathrm{C} / \mathrm{G}$ & 78 & 52 & 76 & 50.7 & \\
\hline $\mathrm{G} / \mathrm{G}$ & 38 & 25.3 & 51 & 34 & \\
\hline $\mathrm{C} / \mathrm{C}$ & 34 & 22.7 & 23 & 15.3 & \\
\hline rs1871054 genotypes & & & & & 0.939 \\
\hline $\mathrm{C} / \mathrm{T}$ & 70 & 46.7 & 67 & 44.7 & \\
\hline $\mathrm{T} / \mathrm{T}$ & 41 & 27.3 & 43 & 28.7 & \\
\hline $\mathrm{C} / \mathrm{C}$ & 39 & 26 & 40 & 26.7 & \\
\hline \multicolumn{6}{|l|}{ MMP13 gene } \\
\hline rs3819089 genotypes & & & & & 0.729 \\
\hline $\mathrm{G} / \mathrm{G}$ & 115 & 76.7 & 111 & 74 & \\
\hline $\mathrm{G} / \mathrm{A}$ & 32 & 21.3 & 34 & 22.7 & \\
\hline$A / A$ & 3 & 2 & 5 & 3.3 & \\
\hline \multicolumn{6}{|l|}{ ADAMTS14 gene } \\
\hline rs4747096 genotypes & & & & & 0.529 \\
\hline $\mathrm{A} / \mathrm{A}$ & 118 & 78.7 & 116 & 77.3 & \\
\hline$A / G$ & 29 & 19.3 & 33 & 22 & \\
\hline $\mathrm{G} / \mathrm{G}$ & 3 & 2 & 1 & 0.7 & \\
\hline
\end{tabular}

were used for the multivariate analysis. Osteoarthritis of the control and patient groups was determined as the dependent variable, while ADAMTS14 rs4747096, ADAM12 rs3740199, MMP13 rs3819089, and ADAM12 rs1871054 were determined as the independent variables. A $p$ value of $<0.05$ was considered statistically significant.

\section{RESULTS}

The demographic data of 300 participants who were included in the study are summarized in Table I. While 76 (11 males, 65 females) of the patients in the patient group showed K-L Grade III OA findings on their direct radiographs, 74 (15 males, 59 females) showed K-L Grade IV OA findings.

\section{TABLE III}

Results of studies investigating the relationship between ADAM12 (rs1871054 and rs3740199) polymorphisms and knee OA

\begin{tabular}{|c|c|c|c|c|}
\hline Study & Population & SNPs & Pathologic findings & Results in sex \\
\hline \multirow[t]{3}{*}{ Kerna et al. (2009) } & Estonian population & rs3740199 & PFKOA & $\mathrm{M}(+)$ \\
\hline & & rs1871054 & PFKOA & $F(-)$ \\
\hline & & & & $\mathrm{M} / \mathrm{F}(-)$ \\
\hline Kerna et al. $(2013)^{*}$ & Estonian population & rs1871054 & Advance stage OA osteophyte formation & $\mathrm{M} / \mathrm{F}(+)$ \\
\hline \multirow[t]{2}{*}{ Wang et al. (2015) } & Chinese Han population & rs3740199 & PFKOA & $\mathrm{M} / \mathrm{F}(-)$ \\
\hline & & rs1871054 & & \\
\hline \multirow[t]{2}{*}{ Lou et al. (2014) } & Chinese Han population & rs3740199 & PFKOA & $\mathrm{M} / \mathrm{F}(-)$ \\
\hline & & rs1871054 & PFKOA susceptibility & $\mathrm{M} / \mathrm{F}(+)$ \\
\hline \multirow[t]{2}{*}{ Poonpet et al. (2016) } & Thai population & rs3740199 & PFKOA susceptibility & $M(+)$ \\
\hline & & & & $F(-)$ \\
\hline
\end{tabular}


The distribution of the genotypes of the SNPs of ADAM12, ADAMTS14, and MMP13 genes conformed to the Hardy-Weinberg equilibrium, and the genotyping success rate was $100 \%$. The frequencies $(\%)$ and statistical comparisons of the SNP genotypes for the study group are presented in Table II. When evaluating the frequency of nucleotides in the alleles in the rs3740199 and rs1871054 genotypes in $A D A M 12$ gene, the most common nucleotides in both the control and patient groups were CG for rs3740199 and CT for rs1871054. On the other hand, when the MMP13 rs3819089 genotype and ADAMTS14 rs4747096 genotype were examined, the most common nucleotides in the alleles were GG for MMP13 rs3819089 and AA for ADAMTS14 rs4747096. To test whether the probability of having OA could be predicted by the existence of MMP13 rs3819089, ADAM12 rs3740199 and rs1871054, and ADAMTS14 rs4747096 genotypes, a binary logistic regression analysis (backward elimination) was conducted, which revealed a statistically insignificant relationship (Table II).

\section{DISCUSSION}

A genetic tendency for developing $\mathrm{OA}$ has been reported as being responsible for 39 to $65 \%$ of OA cases. Although several genes have been studied in OA, the exact mechanism remains to be elucidated. However, the available data strongly suggests that genetic variations may alter the structure or metabolism of the cartilage and bone. Therefore, the relationship between ADAM12 rs3740199 and rs1871054, ADAMTS14 rs4747096, and MMP13 rs3819089 genotypes and the presence of knee $\mathrm{OA}$ in the middle-advanced age population (40 to 75 years) was evaluated.

Although the association of ADAM12 rs1871054 and rs3740199 polymorphisms with OA has been previously investigated, no consensus has been achieved. In the literature, there are numerous research articles and meta-analysis studies investigating the effect of both SNPs of the ADAM12 gene on OA development (Table III). A common finding in the meta-analyses was that ADAM12 rs1871054 was predisposed to knee OA; however, there was no significant relationship between ADAM12 rs3740199 and susceptibility to knee $\mathrm{OA}^{[1,16-19]}$ apart from in a meta-analysis conducted by $\mathrm{Wu}$ et $\mathrm{al}^{\left[{ }^{[20]}\right.}$ where it was suggested that rs3740199 only increased OA vulnerability in males. In this study, no significant relationship was detected between ADAM12 rs3740199 and rs1871054 and advanced-stage knee OA in the Turkish population.
The ADAMTS protease family, which has domains that play a role in the destruction of collagen polysaccharides in the cartilage matrix, is considered a possible genetic factor of OA. Therefore, studies aiming to show the effect of gene polymorphisms on OA pathogenesis in proteases, which are members of the ADAMTS family, have gained importance in recent years. ${ }^{[21]}$ Rodriguez-Lopez et al. ${ }^{[12]}$ examined the polymorphisms of members of the $A D A M$ and ADAMTS family, which may cause OA development by damaging protein function. As a result of this study, there was a significant relationship between the rs4747096 SNP and knee OA predisposition in female patients who underwent total knee replacement surgery due to knee OA. In another study, Poonpet et al. ${ }^{[13]}$ found a relationship between ADAMTS14 rs4747096 and the risk of knee OA in Thai female patients, although no significant association was observed in male patients. In addition, in a study including the Chinese Han population, the rs4747096 SNP increased the risk of knee OA. ${ }^{[6]}$ In our study, there was no significant relationship between advanced-stage knee $\mathrm{OA}$ and the ADAMTS14 rs4747096 SNP. ${ }^{[22]}$

Recent studies on the MMP gene family show that the MMP13 enzyme is responsible for type 2 collagen degradation and that joint cartilage damage, which is caused by an increase in the activity of this enzyme, causes OA. ${ }^{[23]}$ Sathrendra et al. ${ }^{[14]}$ reported that the $r s 3819089$ SNP was protective against nonunion in the Chinese Han population. Our study evaluated the relationship between the MMP13 rs3819089 polymorphism and advanced-stage OA. In this study, no significant relationship was found between the rs3819089 polymorphism and OA.

Although recent studies have shown that these gene polymorphisms are associated with the development of $\mathrm{OA}$ in different populations, we found a significant association in our study. This may be due to different polymorphisms of the same genes affecting the development of OA. Moreover, this difference can be attributed to the width of the cohort examined, the characteristics of the selected control group, the sex and age of the participants, and the severity of the disease. In addition, as each population has its own gene pool and, thus, the genotype distributions and allele frequencies would be different in different ethnic groups. This suggests that ethnicity may be the reason for heterogeneity between studies. The epigenetic factors, including environmental conditions, such as diet, physical activity, and lifestyle, are specific to each population 
and can cause changes in gene-gene interactions. As a result, the genetic effect of polymorphism in a gene may differ between populations.

Although recently, molecular therapies for pathways that play a role in the pathogenesis of inflammatory arthritis have been developed, there is no disease-modifying agent used in OA. One of the main reasons for this is that the pathogenesis of OA is complex, and the pathways that play a role in pathogenesis are essential for cartilage and bone homeostasis. ${ }^{[24]}$ Currently, studies on articular cartilage with an approach to $\mathrm{OA}$ pathogenesis through the pathogenetic concept reveal metalloproteinase-mediated cartilage destruction and the effect of these enzyme genotypes on OA. ${ }^{[1,16-19]} \mathrm{A}$ better understanding of the pathogenesis of OA would pave the way for the development of effective target-specific therapies, which would aid in cartilage repair and reduce cartilage destruction.

Nonetheless, this study has several limitations. First, only cases with Grade III and IV OA according to the K-L staging system were included in the study. Second, it is not possible to make a comparison between sexes due to the unequal number of male and female patients. Including patients with Grade II OA and comparing the results between sexes may have affected the results. Third, only the tibiofemoral joint was evaluated, and signs of arthritis in the patellofemoral joint were not included in the evaluation. Fourth, a clinical functional evaluation was not performed in our study, and only radiological evaluation was used for the evaluation. Fifth, the relationship between other direct radiographic findings of knee OA, such as osteophyte formation and gene polymorphisms, was unable to be evaluated. Finally, the environmental risk factors that patients were exposed to in terms of OA development were not taken into consideration.

In conclusion, the present study results suggest that ADAM12 rs3740199 and rs1871054, MMP13 rs3819089, and ADAMTS14 rs4747096 polymorphisms are not related to advanced-knee OA in the Turkish population. This is the first study to investigate the relationship between the SNPs of ADAM12, ADAMTS14, and MMP13 genes and the development of OA in the Turkish population, and it would contribute to our understanding of the molecular bases of OA. Despite this, it may be possible that other known or unknown SNPs within these genes could still be important in the pathogenesis of OA (i.e., haplotyping of all the SNPs in a candidate gene may be positive). Therefore, further studies are required to explore the usefulness of several gene polymorphisms as markers of disease susceptibility and for risk stratification and to define their precise role in the pathogenesis of OA.

\section{Acknowledgments}

We would like to thank Prof. Ayse Canan Yazıcı Guvercin, $\mathrm{PhD}$ for her valuable assistance with the statistical analysis.

\section{Declaration of conflicting interests}

The authors declared no conflicts of interest with respect to the authorship and/or publication of this article.

\section{Funding}

The authors received no financial support for the research and/or authorship of this article.

\section{REFERENCES}

1. Lv ZT, Liang $S$, Huang XJ, Cheng $\mathrm{P}, \mathrm{Zhu} \mathrm{WT}$, Chen AM. Association between ADAM12 single-nucleotide polymorphisms and knee osteoarthritis: A meta-analysis. Biomed Res Int 2017;2017:5398181.

2. Geng R, Xu Y, Hu W, Zhao H. The association between MMP-1 gene rs1799750 polymorphism and knee osteoarthritis risk. Biosci Rep 2018;38:BSR20181257.

3. Gok K, Cemeroglu O, Cakirbay H, Gunduz E, Acar M, Cetin EN, et al. Relationship between cytosine-adenine repeat polymorphism of ADAMTS9 gene and clinical and radiologic severity of knee osteoarthritis. Int J Rheum Dis 2018;21:821-7.

4. Loughlin J. The genetic epidemiology of human primary osteoarthritis: current status. Expert Rev Mol Med 2005;7:1-12.

5. Deane KD, Demoruelle MK, Kelmenson LB, Kuhn KA, Norris JM, Holers VM. Genetic and environmental risk factors for rheumatoid arthritis. Best Pract Res Clin Rheumatol 2017;31:3-18.

6. Ma S, Ouyang C, Ren S. Relationship between ADAMTS14/ rs4747096 gene polymorphism and knee osteoarthritis in Chinese population. Biosci Rep 2018;38:BSR20181413.

7. Zhao G, Shi J, Xia J. Analysis of the association between $\mathrm{CDH} 2$ gene polymorphism and osteoarthritis risk. Med Sci (Paris) 2018;34 Focus issue F1:105-12.

8. Kerna I, Kisand K, Laitinen P, Tamm AE, Kumm J, Lintrop $\mathrm{M}$, et al. Association of ADAM12-S protein with radiographic features of knee osteoarthritis and bone and cartilage markers. Rheumatol Int 2012;32:519-23.

9. Valdes AM, Doherty M, Spector TD. The additive effect of individual genes in predicting risk of knee osteoarthritis. Ann Rheum Dis 2008;67:124-7.

10. Kryukov GV, Pennacchio LA, Sunyaev SR. Most rare missense alleles are deleterious in humans: implications for complex disease and association studies. Am J Hum Genet 2007;80:727-39.

11. Neuhold LA, Killar L, Zhao W, Sung ML, Warner L, Kulik J, et al. Postnatal expression in hyaline cartilage of constitutively active human collagenase-3 (MMP-13) induces osteoarthritis in mice. J Clin Invest 2001;107:35-44.

12. Rodriguez-Lopez J, Pombo-Suarez M, Loughlin J, Tsezou A, Blanco FJ, Meulenbelt I, et al. Association of a nsSNP in ADAMTS14 to some osteoarthritis phenotypes. Osteoarthritis Cartilage 2009;17:321-7.

13. Poonpet $\mathrm{T}$, Honsawek S, Tammachote N, Kanitnate S, Tammachote R. ADAMTS14 gene polymorphism associated with knee osteoarthritis in Thai women. Genet Mol Res 2013;12:5301-9. 
14. Sathyendra V, Donahue HJ, Vrana KE, Berg A, Fryzel D, Gandhi J, et al.Single nucleotide polymorphisms in osteogenic genes in atrophic delayed fracture-healing: A preliminary investigation. J Bone Joint Surg [Am] 2014;96:1242-8.

15. Peat G, Thomas E, Duncan R, Wood L, Hay E, Croft P. Clinical classification criteria for knee osteoarthritis: performance in the general population and primary care. Ann Rheum Dis 2006;65:1363-7.

16. Chen J, Wu Y, Yu J, Shen J. Association between tumor necrosis factor alpha rs1800629 polymorphism and risk of osteoarthritis in a Chinese population. Braz J Med Biol Res 2018;51:e7311.

17. Hao Z, Li X, Dai J, Zhao B, Jiang Q. Genetic effects of rs3740199 polymorphism in ADAM12 gene on knee osteoarthritis: A meta-analysis. J Orthop Surg Res 2017;12:94.

18. Hu X, Sun G, Wang W. Association of ADAM 12 polymorphisms with the risk of knee osteoarthritis: metaanalysis of 5048 cases and 6848 controls. Rheumatol Int 2017;37:1659-66.
19. Ren K, Ruan Y, Tang J, Jiang X, Sun H, Nong L, et al. Association of ADAM12 gene polymorphisms with knee osteoarthritis susceptibility. Oncotarget 2017;8:77710-21.

20. $\mathrm{Wu} \mathrm{Z}, \mathrm{Xu} \mathrm{XW}, \mathrm{Zhang} \mathrm{XW}$. The association of ADAM12 polymorphism with osteoarthritis susceptibility: A metaanalysis. Ther Clin Risk Manag 2017;13:821-30.

21. Yang CY, Chanalaris A, Troeberg L. ADAMTS and ADAM metalloproteinases in osteoarthritis - looking beyond the 'usual suspects'. Osteoarthritis Cartilage 2017;25:1000-9.

22. Atik OŞ. What are the expectations of an editor from a scientific article? Jt Dis Relat Surg 2020;31:597-8.

23. Mehana EE, Khafaga AF, El-Blehi SS. The role of matrix metalloproteinases in osteoarthritis pathogenesis: An updated review. Life Sci 2019;234:116786.

24. Pulsatelli L, Addimanda O, Brusi V, Pavloska B, Meliconi R. New findings in osteoarthritis pathogenesis: Therapeutic implications. Ther Adv Chronic Dis 2013;4:23-43. 